\title{
Thyroid paraganglioma accompanied by lymph node metastasis or trachea invasion: A case report
}

\author{
XING YU $^{1 *}$, YONG WANG ${ }^{1 *}$, QIUPING XIE ${ }^{1}$, HAICHAO YAN ${ }^{1}$, QUNZI ZHAO $^{1}$, \\ CHENG XIANG $^{1}$, MAOLIN ZHANG ${ }^{1}$, JIN-FAN LI ${ }^{2}$ and PING WANG ${ }^{1}$ \\ Departments of ${ }^{1}$ Thyroid Surgery and ${ }^{2}$ Pathology, The Second Affiliated Hospital, \\ College of Medicine, Zhejiang University, Hangzhou, Zhejiang 310009, P.R. China
}

Received May 25, 2019; Accepted August 18, 2020

DOI: $10.3892 / 01.2020 .12208$

\begin{abstract}
Thyroid paraganglioma (TP) is an uncommon neuroendocrine tumor with potential for misdiagnosis. Case 1 (male; 44 years old) presented with hoarseness for 3 months. A $2.5-\mathrm{cm}$ gray nodule in the left thyroid was diagnosed as TP by immunohistochemistry. Lymph node metastasis was confirmed by H\&E staining and immunohistochemistry. The patient was followed-up for 3 years without any signs of recurrence or metastasis. Case 2 (female; 39 years old) presented with an asymptomatic but rapidly growing thyroid nodule. The patient underwent thyroidectomy 8 years ago (2008) and was diagnosed with TP postoperatively. The patient was diagnosed with recurrence and exhibited trachea invasion. Thyroidectomy and partial trachea resection were performed. A literature review revealed that 62 other cases of TP have been reported, and most of them exhibited low malignant potential. However, the two cases presented in the current case report exhibited a high malignant potential, accompanied by lymph node metastasis or trachea invasion, and these features imply that the choice of treatment for patients should be based on their different degrees of malignancy.
\end{abstract}

\section{Introduction}

Thyroid paraganglioma (TP) is an uncommon neuroendocrine tumor that arises from the inferior laryngeal paraganglia (1).

Correspondence to: Dr Ping Wang, Department of Thyroid Surgery, The Second Affiliated Hospital, College of Medicine, Zhejiang University, 88 Jiefang Road, Hangzhou, Zhejiang 310009, P.R. China

E-mail: p.wang@zju.edu.cn

Dr Jin-fan Li, Department of Pathology, The Second Affiliated Hospital, College of Medicine, Zhejiang University, 88 Jiefang Road, Hangzhou, Zhejiang 310009, P.R. China

E-mail: jinfli2004@sina.com

*Contributed equally

Key words: thyroid paraganglioma, neuroendocrine tumor, immunohistochemistry, lymph nodes, trachea invasion
One hypothesis is that the inferior laryngeal paraganglia may form within the thyroid capsule, which could eventually develop into an intrathyroid paraganglioma (2). Another hypothesis is that paraganglioma develops from the inferior laryngeal paraganglia, pulls downward slowly, and eventually rests lateral to the thyroid gland (3).

The diagnosis of TP is challenging, not due to its low prevalence (only 60 cases have been reported), but the cytologic and histopathologic similarities with other thyroid tumors, such as medullary thyroid carcinoma (MTC) $(4,5)$. The examination of immunohistochemical staining serves an important role in the definitive diagnosis; however, to the best of our knowledge, the malignant potential of TP remains unknown $(3,6,7)$. Since it was described by Van Miert (8) in 1964, TP has been commonly considered to have low malignant potential $(1,9,10)$.

In the present study, two cases of TP are presented, both of which tended to mimic MTC, one was accompanied by lymph node metastasis, and the other exhibited high malignant potential of trachea invasion. Furthermore, a clinical strategy of positive diagnostic and therapeutic management was introduced with the guidance of a systematic review.

\section{Case report}

Case 1. A 44-year-old man presented with a 3-month history of hoarseness, but without any signs of breathing or swallowing difficulties. The patient was admitted to The Second Affiliated Hospital of Zhejiang University (Hangzhou, China) on September 26, 2016. A 2.6-cm hypoechoic, noncalcific neoplasm in the left thyroid lobe was identified in the ultrasound examination. Serum thyroid stimulating hormone, T3 and T4 were consistent with euthyroid state and serum calcitonin was normal. A fine needle aspiration biopsy (FNAB) was performed, and cytological results were regarded as suspicious for malignancy according to the Bethesda classification system $(11,12)$. During the surgical procedure, a $2.5-\mathrm{cm}$ gray nodule was detected in the thyroid capsule and was presumptively diagnosed as MTC by frozen section analysis. The patient underwent total thyroidectomy and bilateral central neck dissection. In the examination of definitive histopathology, TP was diagnosed by histopathologic and immunohistochemistry features. Tumor cells appeared to be nesting and exhibited a 'zellballen' growth pattern in H\&E staining, and were 
positive for Cg A, Syn, neuronal specific enolase (NSE), Ki67, S-100, CD34, CD31 and p53, and negative for thyroglobulin, thyroid transcription factor 1 (TTF-1), calcitonin, CEA, cytokeratin $(\mathrm{CK})(\mathrm{AE} 1 / 3), \mathrm{CD} 45, \mathrm{Bcl} 2$, inhibin and galectin-3. Among them, the most representative characteristics were analyzed and exhibited in Fig. 1. In addition, the dissected lymph nodes were assessed and metastasis was confirmed by H\&E staining and immunohistochemistry (Figs. 2 and 3). The patient was treated with radiation after the surgery and was in a good condition without any evidence of other metastases during the follow-up (twice a year) at 3 years postoperatively.

Case 2. A 39-year-old woman was suspected to have TP recurrence following hemi-thyroidectomy surgery. The patient was admitted to The Second Affiliated Hospital of Zhejiang University on April 17, 2016. The patient was diagnosed with TP during the last hemithyroidectomy surgery performed 8 years ago (2008), based on the typical histopathologic and immunohistochemical characteristics as reported in a previous article (5). Postoperatively, the patient was doing well without any sign of recurrence or metastasis, and no other relevant finding was found during the follow-up (twice a year) for 7 years. However, a $2.5-\mathrm{cm}$ hypoechoic nodule was identified by physical and ultrasound examination in the 'thyroid area' 1 year ago (2015). According to the computed tomography examination, the tumor exhibited aggressive potential with trachea invasion (Fig. 4A). For radical resection, thyroidectomy and trachea incision were performed. All tumor tissues were completely dissected, and a temporary cannula was indwelling in the channel of trachea (Fig. 4B). Immunohistochemical staining was positive for $\mathrm{Cg} \mathrm{A}$, Syn, NSE and CD56 in tumor cells, negative for thyroglobulin, TTF-1, calcitonin, CEA, CK (AE1/3) and P53 in tumor cells, and $20 \%$ positive for $\mathrm{S}-100$ in sustentacular cells. Furthermore, gene analysis was performed, and there was no mutation in the genes of Kras exon 2/3/4, NRAS proto-oncogene, GTPase exon 2/3/4, telomerase reverse transcriptase C228T/T250T, phosphatidylinositol-4,5-bisphosphate 3-kinase catalytic subunit $\alpha$ E545K/H1047R and BRAF V600E. In addition, the right thyroid lobe was confirmed to be normal, and no metastatic lymph node was found in the neck VI area ( $0 / 10$ positive). The patient was diagnosed with recurring TP and treated with radiation (8). The patient was follow-up at the outpatient twice a year, and no sign of recurrence or metastasis 4 years postoperatively.

$H \& E$ and immunohistochemical staining. Surgical specimens were fixed in $10 \%$ phosphate-buffered, neutral formaldehyde solution at room temperature for $24 \mathrm{~h}$. Tissue sections $(4-\mu \mathrm{m}$ thick) were deparaffinized in a descending alcohol series, rehydrated (using $10 \mathrm{mM}$ sodium citrate, $\mathrm{PH} 8.5$, at $80^{\circ} \mathrm{C}$ for $30 \mathrm{~min}$ ), and exposed to heat-induced antigen retrieval for $5 \mathrm{~min}$ in an autoclave at $121^{\circ} \mathrm{C}$ in $\mathrm{pH} 7.8$ Tris-EDTA-Citrate buffer. $4-\mu \mathrm{m}$ sections were stained with $\mathrm{H} \& \mathrm{E}$ and immunohistochemistry, respectively. $H \& E$ staining: Incubate the slides with hematoxylin solution in a staining jar for $10 \mathrm{~min}$ to stain the nuclei at room temperature. Transfer the slides to a staining jar with running water (tap water is fine) till the water is clear. Transfer the slides to a staining jar with Eosin solution for $3 \mathrm{~min}$ at room temperature. Then transfer the slides into staining jars with $70 \%$ ethanol for $20 \mathrm{sec}, 90 \%$ ethanol for $20 \mathrm{sec}, 100 \%$ ethanol for $1 \mathrm{~min}$ and xylene for $3 \mathrm{~min}$. Take out slides from xylene and place the slides in a fume hood till the slides are dry. Store the slides at room temperature. $\mathrm{H} \& \mathrm{E}$ images were captured with a 14.0 MP digital microscope camera which is attached via a c-mount to the side port of a Leica DM 2500 microscope (for the light, magnifications: $x 40$, $\mathrm{x} 100$, x200, and x400). Immunohistochemistry (IHC) staining: Place the slides in a wet chamber (with $0.3 \%$ nonfat dry milk) and block the sections with blocking buffer for at least $30 \mathrm{~min}$ at room temperature. Then incubated at room temperature for $2 \mathrm{~h}$ in a humid chamber. Primary antibody was applied at $37^{\circ} \mathrm{C}$ for $60 \mathrm{~min}$. The specific antibodies included TTF-1 (8G7G3/1; 1:100 dilution), calcitonin (GA51561-2; 1:100 dilution), CEA (IS62230-2; 1:400 dilution), NSE (M0873; 1:100 dilution), Syn (GA660; 1:400 dilution), Cg A (M0869; 1:100 dilution), S-100 (GA504; 1:400 dilution) (4), Ki67 (M7240; 1:100 dilution) (5), CK (AE1/3) (IS053; 1:100 dilution), thyroglobulin (A0251; 1:200 dilution) (7), p53 (GA616; dilution 1:200) (10), galectin-3 (M3/38; 1:100 dilution) and Bcl2 (M0887; 1:400 dilution) (13) (Agilent Technologies, Inc.). Subsequently the sections were washed three times with PBS and anti-mice/rabbit enzyme-labeled secondary antibodies (P0447 or E0432; 1:200 dilution, Dako; Agilent Technologies, Inc.) were then applied at room temperature for $15 \mathrm{~min}$. IHC images were captured with a 14.0 MP digital microscope camera which is attached via a c-mount to the side port of a Leica DM 2500 microscope (magnifications: x40, x100, x200 and x400). Immunohistochemistry was performed according to the DAKO EnVision method $(13,14)$. The expression levels of TTF-1, calcitonin, CEA, NSE, Syn, Cg A, S-100, Ki67, CK (AE1/3), thyroglobulin, p53, galectin-3 and $\mathrm{Bcl} 2$ were semi-quantified using a visual grading system based on the extent of staining as previously described (14): Grade 0, virtually no immunoreactivity; grade 1, patchy to diffuse weak immunoreactivity; grade 2 , patchy to diffuse moderate immunoreactivity; and grade 3 , patchy to diffuse strong immunoreactivity. According to the results of the immunohistochemistry analysis, grade 0 cases were classified as the negative group, and grade 1-3 cases as the positive group. Subsequently the immunohistochemical results were collected and analyzed (Table I).

\section{Discussion}

TP is rare. A detailed literature search [inclusion criteria (Thyroid[Title/Abstract]) AND (Paraganglioma[Title]); Database NCBI (https://pubmed.ncbi.nlm.nih.gov)] of TPs between 1975 and June 30, 2019 was performed. Excluding the diagnosis of carotid body paraganglioma, jugular paraganglioma, medullary thyroid carcinoma, and the repeated reported cases, 62 reported TP cases were included from 124 related studies (Fig. 5). TP represents a challenge for surgeons in terms of diagnosis and making appropriate treatment decisions (15). Among them, the malignant potential of TPs remains controversial, and more studies are required to help surgeons make diagnostic and therapeutic management decisions. Due to the rarity of this disease, samples from every available case need to be collected to validate the findings.

The examination of immunohistochemistry staining serves an important role in the definitive diagnosis (5). In 


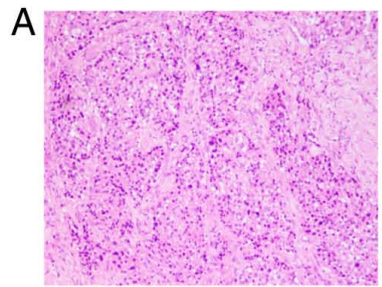

$H \& E$

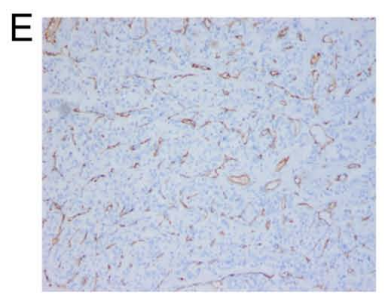

CD 31

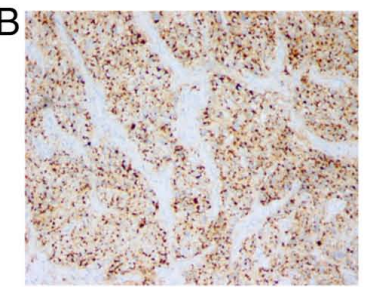

$\operatorname{Cg} A$

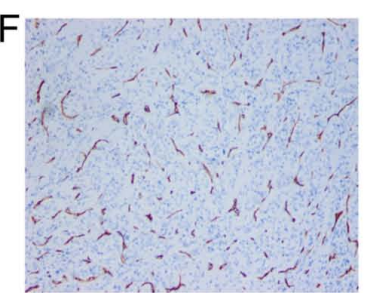

CD 34

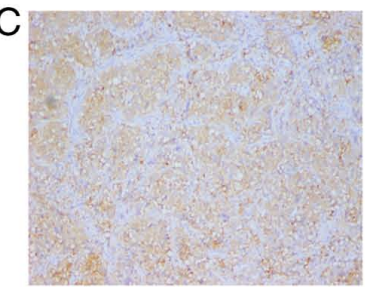

NSE

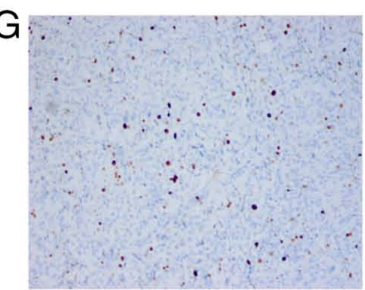

$\mathrm{KI}-67$

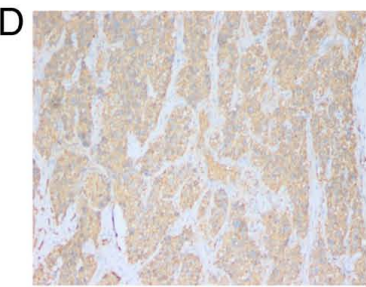

Syn

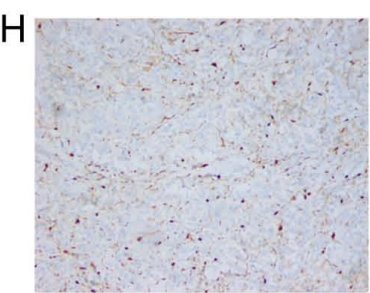

S 100

Figure 1. Typical pathologic and immunohistochemical features of thyroid paraganglioma (case 1). (A) Neoplasm is surrounded by thin fibrous capsules and chief cells arranged in nests ('zellballen' architecture). H\&E. Magnification, x100. (B) Chief cells showing strong positivity in CgA immunostaining. Magnification, x100. (C) Strongly positive NSE staining. Magnification, x100. (D) Positive Syn staining. Magnification, x100. Endothelial cells exhibiting positivity for (E) CD31 and (F) CD34. Magnification, x100. (G) Chief cells showing weak positivity for Ki67. Magnification, x100. (H) Sustentacular cells exhibiting positivity for S-100. Magnification, x100. NSE, neuron specific enolase.
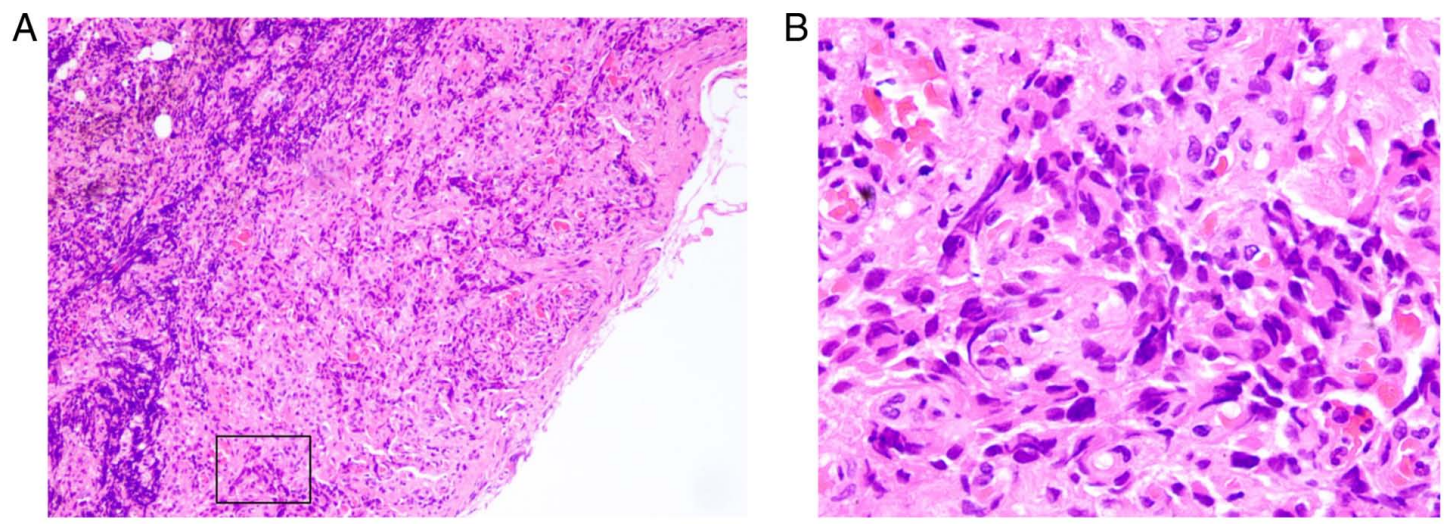

Figure 2. Pathologic characteristics of lymph nodes and neoplasm metastasis in TP (case 1). (A) Neoplasms were surrounded by fibrous capsules and plasma cells. H\&E staining. Magnification, x40. $\square$ presents the magnified area. (B) Chief cells were surrounded by fiber cells and arranged in nests ('zellballen' architecture). H\&E staining. Magnification, x400.
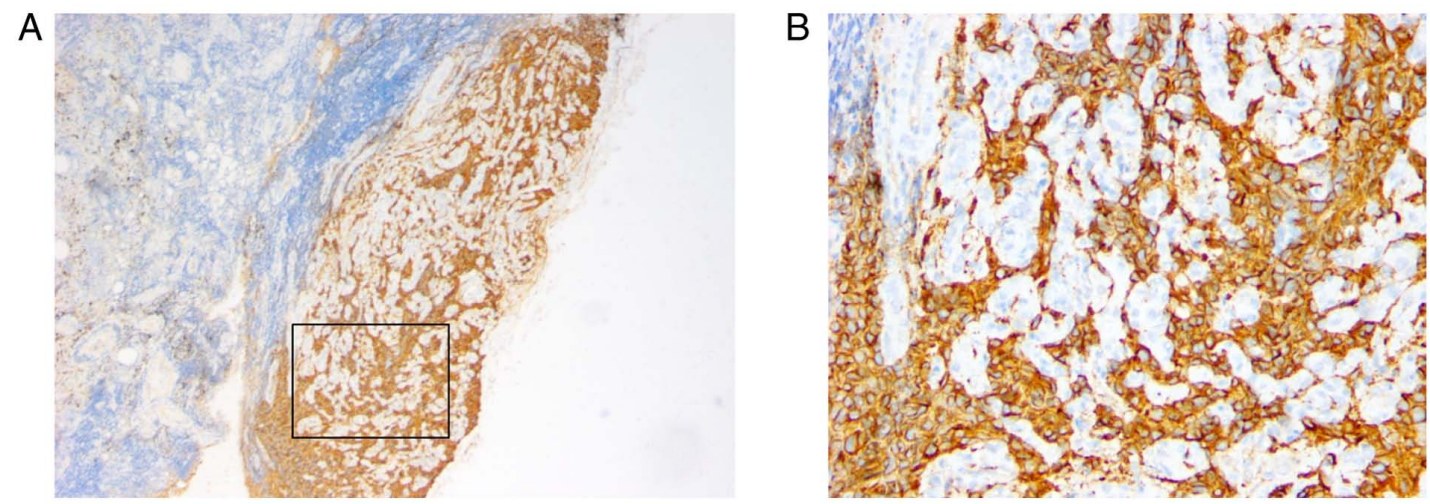

Figure 3. Immunohistochemical features of lymph nodes and neoplasm metastasis in TP (case 1). (A) Neoplasms exhibiting strong positivity in Syn immunostaining, while negativity was observed in the physical lymphoid tissues. Magnification, x40. $\square$ presents the magnified area. (B) Syn staining was strongly positive in chief cells, while it was negative in fiber cells. Magnification, x200.

the present study, tissues were obtained from two patients for diagnostic purposes. Several typical characteristics of the two cases are recorded in Table I, and the results were consistent with the World Health Organization's classification 
A

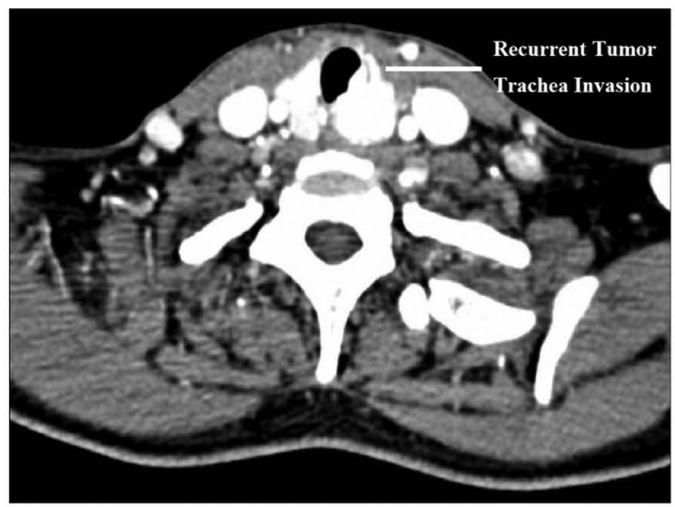

B

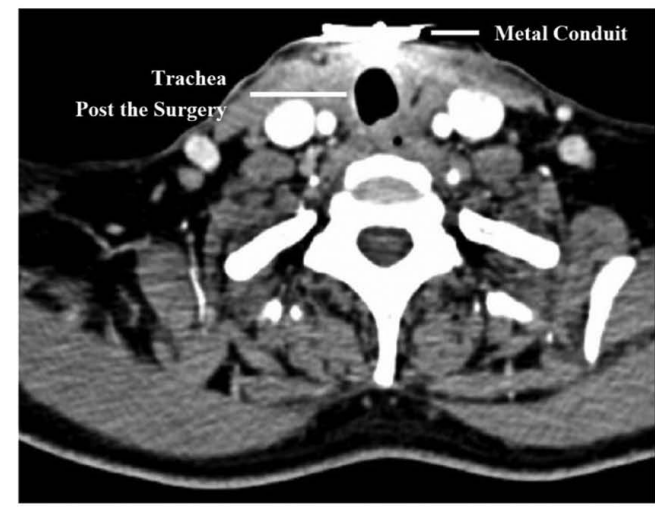

Figure 4. Computed tomography results before and after surgery (case 2). (A) Recurrent tumor was identified in the 'thyroid area', and trachea invasion was observed. (B) All tumor tissues were completely dissected.

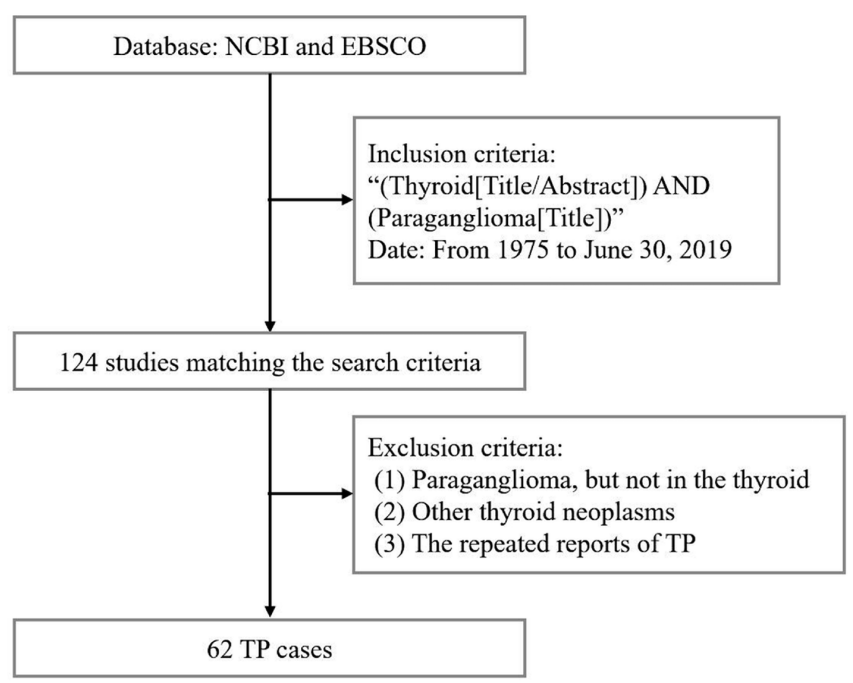

Figure 5. Flowchart of literature review. Cases of thyroid paraganglioma unti June 30, 2019 were searched, recorded and analyzed using the NCBI and EBSCO databases. NCBI, National Center for Biotechnology Information; TP, thyroid paraganglioma.

of endocrine tumors for paraganglioma (16). The molecular features of TPs have been presented and summarized in previous studies (3-5). Based on the studies identified in the literature analysis, chief cells exhibit strong positivity for the markers of neuroendocrine tumors, including $\mathrm{Cg} \mathrm{A}$ (positive rate, 100\%), NSE (positive rate, 100\%) and Syn (positive rate, $100 \%$ ). The negative makers include calcitonin (negative rate, 100\%), CK (AE1/3) (negative rate, 100\%) and CEA (negative rate, $100 \%$ ). The thyroid originated markers also exhibit negative staining, including thyroglobulin (negative rate, $97.1 \%$ ) and TTF-1 (negative rate, 96.4\%). In addition, S- 100 staining was positive in sustentacular cells (positive rate, 97.8\%; Fig. 6A). Therefore, a wide range of antibodies should be examined for the definitive diagnosis of TP, including at least Cg A, NSE, Syn, calcitonin, CEA, CK (AE1/3), thyroglobulin and TTF-1 in tumor cells, and S-100 in sustentacular cells.

However, a definitive diagnosis of TP always requires the examination of immunohistochemistry postoperatively, while it is rarely diagnosed preoperatively or intraoperatively (15). The diagnosis of TP is difficult due to its overlapping cytologic features, and it is often misdiagnosed as benign or malignant thyroid tumors (16). As shown in the present two cases, the unique molecular markers of TP compared with other thyroid neoplasms include positive expression of Cg A, NSE, Syn and S-100, and negative expression of calcitonin, CK (AE1/3), CEA, thyroglobulin and TTF-1. To determine the mechanisms of TP, the embryological origin needs to be investigated more thoroughly.

TP is supposed to be derived from the inferior laryngeal paraganglion and then migrate into the thyroid lobe with embryologic development (17). This embryologic origin explains that the cytologic characteristics of TP are similar to other neuroendocrine lesions of the thyroid, including $\mathrm{C}$-cell tumors (C-cell hyperplasia and MTC) and follicular-derived tumors (follicular and papillary thyroid carcinoma) $(5,16)$. Therefore, it is difficult to diagnose TP only by distinguishing cytologic features, such as FNAB, frozen section analysis or general pathology.

Through the literature review, 39 TP cases were performed with frozen section analysis intraoperatively, and the results were collected and summarized. Only eight patients were diagnosed correctly; however, most patients were misdiagnosed as having MTC, FTC or secondary metastatic tumors from neuroendocrine carcinoma (Fig. 6B). A comprehensive examination of CT, MRI and serum proteins needs to be performed prior to biopsy. The biomarkers thyroglobulin, calcitonin and catecholamine are specific for other thyroid neoplasms, and can help to distinguish TP. Elevated thyroglobulin levels indicate a diagnosis of papillary thyroid carcinoma or follicular thyroid carcinoma (18). Furthermore, elevated calcitonin levels are a specific characteristic of MTC (19). Catecholamine hypersecretion can be occasionally detected in malignant paraganglioma, defined by distant metastases or extensive local invasion into adjacent tissue (20). However, as reported in the present study and previous literature (4-6), the levels of catecholamine were not increased in TP, which can be differentiated from other paragangliomas. Similarly, a correct diagnosis could not be established preoperatively by FNAB, except for by immunohistochemistry, until the introduction of the novel method using cell block material (21). The cell block method made it possible to investigate the pathological characteristic of the 'zellballen' pattern in FNAB specimen preoperatively. Additionally, it could be evaluated by immunohistochemistry, which was consistent with the characteristics 
Table I. Immunohistochemical staining results of the two cases of thyroid paraganglioma.

\begin{tabular}{lll}
\hline Immunohistochemistry & \multicolumn{1}{c}{ Case 1} & Case 2 \\
\hline Tumor cells, chromogranin A & Positive & Positive \\
Tumor cells, NSE & Positive & Positive \\
Tumor cells, synaptophysin & Positive & Positive \\
Tumor cells, calcitonin & Negative & Negative \\
Tumor cells, cytokeratin & Negative & Negative \\
Tumor cells, thyroglobulin & Negative & Negative \\
Tumor cells, TTF-1 & Negative & Negative \\
Tumor cells, calcitonin & Negative & Negative \\
Tumor cells, CEA & Negative & Negative \\
Tumor cells, CK $($ AE1/3) & Negative & Negative \\
Tumor cells, P53 & Weakly positive & Negative \\
Tumor cells, CD56 & Not performed & $20 \%$ positive \\
Endothelial cells, CD34 & Positive & Not performed \\
Endothelial cells, CD31 & Positive & Not performed \\
Tumor cells, Ki67 & $10 \%$ positive & $10 \%$ positive \\
Sustentacular cells, S-100 & Positive & $20 \%$ positive
\end{tabular}

NSE, neuronal specific enolase; TTF-1, thyroid transcription factor 1; CEA, carcinoembryonic antigen; CK, cytokeratin.

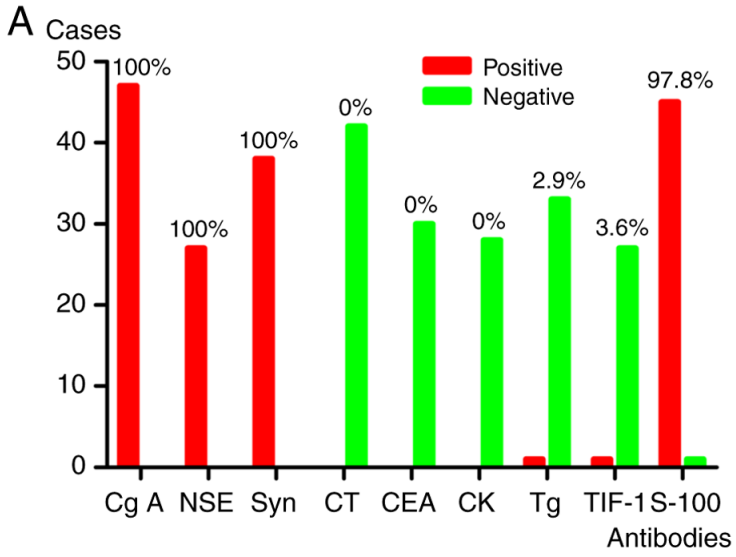

B

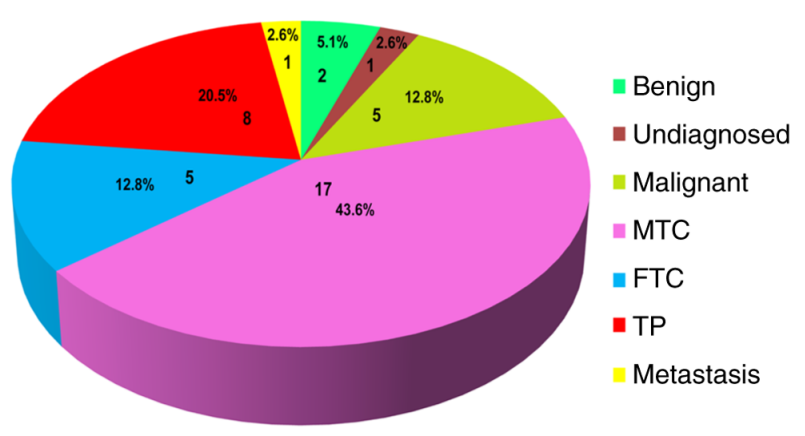

Figure 6. Summary of pathological and immunohistochemical characteristics of TP. (A) Positive and negative rates of several typical markers assessed by immunohistochemistry. The positive percentages of each immunohistochemical characteristics were labeled. (B) Through the literature review, 39 TP cases were performed with frozen section analysis intraoperatively, and the preliminarily results were summarized. NSE, neuron specific enolase; CT, calcitonin; CK, cytokeratin (AE1/3); Tg, thyroglobulin; MTC, medullary thyroid carcinoma; FTC, follicular thyroid carcinoma; TP, thyroid paraganglioma.

of paraganglioma described previously $(16,21)$. The diagnosis of TP can be successfully achieved, and these observations suggest that the method comprising FNAB and immunohistochemistry is promising for the differentiation between TP and other thyroid neoplasms.

Different options that range between subtotal thyroidectomy and total thyroidectomy are recommended depending on the malignancy of neoplasms, which is based on the presence of metastasis to lymph nodes or other organs $(4,5)$. Compared with other thyroid neoplasms, adjuvant therapies for TPs are controversial due to most of them presenting as mild and limited germination (15). However, the malignant type of TPs with local infiltration, and lymph node or distant metastasis, still requires attention (3). In the present study, a patient (case 1) was diagnosed with TP accompanied by lymph node metastasis. Another implication is recurrence of TP that probably represented high malignant potential. As shown in case 2, the patient underwent hemithyroidectomy and was followed-up for 7 years until recurrence. The tumor exhibited malignant behaviors of rapid growth and trachea invasion in the first recurring year. For these patients, radiotherapy was helpful, and was performed after total thyroidectomy and bilateral central node dissection (8). Both patients in the present study underwent standard treatment for TP with favorable outcomes $(2-5,8)$. According to these experiences, thyroidectomy is appropriate in the treatment of TP $(4,5)$. Since some cases of TP exhibit highly malignant behavior, radiotherapy may be considered as a second-line treatment (8). The duration of follow-up should be $>3$ years due to the possibility of recurrence. 
In conclusion, these two rare cases are helpful to recognize the malignant potential of TP. The present study is helpful to create a standardized set of immunohistochemical staining markers to completely distinguish TP from other thyroid neoplasms. Besides local infiltration, distant metastasis, manifestations of recurrence and lymph node metastasis may be other symptoms representing high malignant potential. The appropriate therapeutic management requires verification in further clinical trials, and methods of surgical dissection or radiotherapy depend on the malignant potential of the tumor.

\section{Acknowledgements}

Not applicable.

\section{Funding}

The present study was supported by the Public Welfare Projects of Zhejiang Province (grant no. LGH18H160002).

\section{Availability of data and materials}

The datasets used and/or analyzed during the present study are available from the corresponding author upon reasonable request.

\section{Authors' contributions}

PW and JFL assessed the clinical findings of the cases and were responsible for the conception of the present study. XY, YW and QX designed the research and were responsible for quality control of the data. HY, QZ, CX and MZ made substantial contributions to acquisition, analysis and interpretation of the data. XY and YW drafted the initial manuscript. PW and JFL provided constructive discussions and revised the manuscript critically for important intellectual content. All authors have read and approved the final manuscript.

\section{Ethics approval and consent to participate}

The present study was approved by the Ethics Committee of the 2nd Affiliated Hospital, School of Medicine, Zhejiang University (Hangzhou, China; approval no. 2019-393), and written informed consent was provided by all patients.

\section{Patient consent for publication}

Not applicable.

\section{Competing interests}

The authors declare that they have no competing interests.

\section{References}

1. Lee SM and Policarpio-Nicolas ML: Thyroid paraganglioma. Arch Pathol Lab Med 139: 1062-1067, 2015.
2. Phitayakorn R, Faquin W, Wei N, Barbesino G and Stephen AE: Thyroid-associated paragangliomas. Thyroid 21: 725-733, 2011.

3. Navaratne L, Mathew RG, Kousparos G and McCombe A: The management of locally invasive primary thyroid paraganglioma: A case report and review of the literature. Head Neck Pathol 11: 139-145, 2016.

4. Pelizzo MR, Conti C, Pennelli G, Bellan E, Cook GJ, Wong KK, Colletti PM, Boschin IM and Rubello D: Thyroid paraganglioma: Our experience and systematic review of the literature on a rare tumor. Am J Clin Oncol 41: 416-423, 2016.

5. Yu X, Wang Y, Wang P, Ji CH, Miao CD and Zheng S: Primary thyroid paraganglioma mimicking medullary thyroid carcinoma: A case report. Oncol Lett 10: 1000-1002, 2015.

6. Bugalho MJ, Silva AL and Domingues R: Coexistence of paraganglioma/pheochromocytoma and papillary thyroid carcinoma: A four-case series analysis. Fam Cancer 14: 603-607, 2015.

7. Armstrong MJ, Chiosea SI, Carty SE, Hodak SP and Yip L: Thyroid paragangliomas are locally aggressive. Thyroid 22 : 88-93, 2012.

8. Vanmiert PJ: The treatment of chemodectomas by radiotherapy. Proc R Soc Med 57: 946-951, 1964.

9. Kieu V, Yuen A, Tassone P and Hobbs CG: Cervical paraganglioma presenting as thyroid neoplasia. Otolaryngol Head Neck Surg 146: 516-518, 2012

10. Castelblanco E, Gallel P, Ros S, Gatius S, Valls J, De-Cubas AA, Maliszewska A, Yebra-Pimentel MT, Menarguez J, Gamallo C, et al: Thyroid paraganglioma. Report of 3 cases and description of an immunohistochemical profile useful in the differential diagnosis with medullary thyroid carcinoma, based on complementary DNA array results. Hum Pathol 43: 1103-1112, 2012.

11. Lim JXY, Nga ME, Chan DKH, Tan WB, Parameswaran R and Ngiam KY: Subclassification of Bethesda atypical and follicular neoplasm categories according to nuclear and architectural atypia improves discrimination of thyroid malignancy risk. Thyroid 28: 511-521, 2018.

12. Kim M, Jeon MJ, Han M, Lee JH, Song DE, Baek JH, Kim TY, Kim WB, Shong YK and Kim WG: Tumor growth rate does not predict malignancy in surgically resected thyroid nodules classified as Bethesda category III with architectural atypia. Thyroid 29: 216-221, 2018.

13. Bockhorn M, Sheu SY, Frilling A, Molmenti E, Schmid KW and Broelsch CE: Paraganglioma-like medullary thyroid carcinoma: A rare entity. Thyroid 15: 1363-1367, 2005.

14. He Y, Zhou Y, Zhang J, Yuan F, Wang J, Du L, Zhou Q, Liang J and Ding X: Tumor immunohistochemistry and preoperative magnetic resonance imaging features predict local recurrence of giant cell tumor of bone following intralesional curettage. Oncol Lett 17: 1425-1434, 2019.

15. von Dobschuetz E, Leijon H, Schalin-Jäntti C, Schiavi F, Brauckhoff M, Peczkowska M, Spiazzi G, Demattè S, Cecchini ME, Sartorato P, et al: A registry-based study of thyroid paraganglioma: Histological and genetic characteristics. Endocr Relat Cancer 22: 191-204, 2015.

16. Williams MD and Tischler AS: Update from the 4th edition of the world health organization classification of head and neck tumours: Paragangliomas. Head Neck Pathol 11: 88-95, 2017.

17. Taweevisit M, Bunyayothin W and Thorner PS: Thyroid paraganglioma: 'Naked' nuclei as a clue to diagnosis on imprint cytology. Endocr Pathol 26: 232-238, 2015.

18. Lin JD: Thyroglobulin and human thyroid cancer. Clin Chim Acta 388: 15-21, 2008.

19. Emmertsen K: Medullary thyroid carcinoma and calcitonin. Dan Med Bull 32: 1-28, 1985.

20. Fishbein L: Pheochromocytoma and paraganglioma: Genetics, diagnosis, and treatment. Hematol Oncol Clin North Am 30: 135-150, 2016.

21. Çetin S, Kir G and Yilmaz M: Thyroid paraganglioma diagnosed by fine-needle aspiration biopsy, correlated with histopathological findings: Report of a case. Diagn Cytopathol 44: 643-647, 2016.

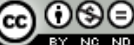

This work is licensed under a Creative Commons Attribution-NonCommercial-NoDerivatives 4.0 International (CC BY-NC-ND 4.0) License. 